\title{
Touchard-Riordan formulas, $T$-fractions, and Jacobi's triple product identity
}

\author{
Matthieu Josuat-Vergès ${ }^{1}{ }^{\dagger}$ and Jang-Soo Kim ${ }^{2} \ddagger$ \\ ${ }^{1}$ Fakultät für Mathematik, Universität Wien, Austria \\ ${ }^{2}$ School of Mathematics, University of Minnesota, MN, USA
}

\begin{abstract}
We give a combinatorial proof of a Touchard-Riordan-like formula discovered by the first author. As a consequence we find a connection between his formula and Jacobi's triple product identity. We then give a combinatorial analog of Jacobi's triple product identity by showing that a finite sum can be interpreted as a generating function of weighted Schröder paths, so that the triple product identity is recovered by taking the limit. This can be stated in terms of some continued fractions called $T$-fractions, whose important property is the fact that they satisfy some functional equation. We show that this result permits to explain and generalize some Touchard-Riordan-like formulas appearing in enumerative problems. Résumé. Nous donnons une preuve combinatoire d'une formule à la Touchard-Riordan due au premier auteur. En conséquence, nous faisons apparaître un lien entre cette formule et l'identité du produit triple de Jacobi. Nous donnons un analogue combinatoire à l'identité du produit triple en montrant qu'une somme finie peut être interprétée comme fonction génératrice de chemins de Schröder pondérés, de sorte que l'identité du produit triple s'obtient en passant à la limite. Ceci peut être énoncé en termes de fractions continues appelées $T$-fractions, dont la propriété importante est le fait qu'elle satisfont certaines équations fonctionnelles. Nous montrons que ce résultat permet d'expliquer et généraliser certaines formules à la Touchard-Riordan apparaissant dans des problèmes d'énumération.
\end{abstract}

Keywords: Jacobi's triple product identity, continued fractions, enumeration

\section{Introduction}

\subsection{Touchard-Riordan formulas}

The original result of Touchard [Tou52], later given more explicitly by Riordan [Rio75], answers the combinatorial problem of counting chord diagrams according to the number of crossings. It has also been stated in terms of a continued fraction by Read [Rea79], so that the Touchard-Riordan formula is:

$$
\left[z^{n}\right]\left(\frac{1}{1}-\frac{[1]_{q} z}{1}-\frac{[2]_{q} z}{1}-\cdots\right)=\frac{1}{(1-q)^{n}} \sum_{k=0}^{n}\left(\left(\begin{array}{c}
2 n \\
n-k
\end{array}\right)-\left(\begin{array}{c}
2 n \\
n-k-1
\end{array}\right)\right)(-1)^{k} q^{\left(\begin{array}{c}
k+1 \\
2
\end{array}\right)}
$$

\footnotetext{
${ }^{\dagger}$ Supported by French National Research Agency ANR, grant ANR08-JCJC-0011, and Austrian Science foundation FWF, START grant Y463.

${ }^{\ddagger}$ Supported by French National Research Agency ANR, grant ANR08-JCJC-0011.

1365-8050 (c) 2011 Discrete Mathematics and Theoretical Computer Science (DMTCS), Nancy, France
} 
where $\left[z^{n}\right]$ is the operator that extracts the coefficient of $z^{n}$ in a series, $[n]_{q}$ denotes $\left(1-q^{n}\right) /(1-q)$, and we use the notation for continued fractions as in (10). Recently, several variants have been derived. In particular, using continued fractions and basic hypergeometric series, the first author [JV10] proved the following formula in a slightly different form related with enumeration of alternating permutations:

$$
\left[z^{n}\right]\left(\frac{1}{1}-\frac{[1]_{q}^{2} z}{1}-\frac{[2]_{q}^{2} z}{1}-\cdots\right)=\frac{1}{(1-q)^{2 n}} \sum_{k=0}^{n}\left(\left(\begin{array}{c}
2 n \\
n-k
\end{array}\right)-\left(\begin{array}{c}
2 n \\
n-k-1
\end{array}\right)\right) q^{k(k+1)} \sum_{i=-k}^{k}(-q)^{-i^{2}}
$$

In the first part of this paper, we prove (2) combinatorially. To do this we introduce a combinatorial model whose weight sum is equal to $\sum_{i=-k}^{k}(-q)^{-i^{2}}$. As a consequence of the combinatorial proof we can let $k \rightarrow \infty$ and obtain

$$
\prod_{i \geq 1} \frac{1-q^{i}}{1+q^{i}}=\sum_{i=-\infty}^{\infty}(-q)^{i^{2}}
$$

which is known as the special case $(y=-1)$ of Jacobi's triple product identity:

$$
\prod_{n \geq 1}\left(1-q^{2 n}\right)\left(1+y q^{2 n-1}\right)\left(1+y^{-1} q^{2 n-1}\right)=\sum_{n=-\infty}^{\infty} y^{n} q^{n^{2}}
$$

\subsection{Jacobi's triple product identity}

Jacobi's triple product identity (JTP) is ubiquitous in various areas of mathematics and especially in analytical number theory. Quite a lot of different proofs, generalizations and variants are known, see for example [AB04, Ber06, Sch05, War05] and lots of references therein. Some classical particular cases or consequences are :

$$
\prod_{i \geq 1}\left(1-q^{i}\right)=\sum_{j=-\infty}^{\infty}(-1)^{j} q^{\frac{j(3 j-1)}{2}}
$$

which is known as Euler's pentagonal number theorem, and also (3) and

$$
\prod_{i \geq 1}\left(1-q^{i}\right)^{3}=\sum_{j=0}^{\infty}(-1)^{j}(2 j+1) q^{\frac{j(j+1)}{2}} .
$$

See [Ber06, Chapter 1] for a general reference about these identities.

Now we state the main result.

Theorem 1.1. We define $[n]_{y, q}=\left(1+y q^{n}\right) /(1-q)$. Then

$$
\begin{array}{r}
{\left[z^{n}\right]\left(\frac{1}{1}-\frac{[1]_{y, q}[1]_{y^{-1}, q} z}{1}-\frac{[2]_{q}^{2} z}{1}-\frac{[3]_{y, q}[3]_{y^{-1}, q} z}{1}-\frac{[4]_{q}^{2} z}{1}-\ldots\right)} \\
=\frac{1}{(1-q)^{2 n}} \sum_{k=0}^{n}\left(\left(\begin{array}{c}
2 n \\
n-k
\end{array}\right)-\left(\begin{array}{c}
2 n \\
n-k-1
\end{array}\right)\right) q^{k(k+1)} \sum_{i=-k}^{k} y^{i} q^{-i^{2}}
\end{array}
$$


Note that if $y=-1$, we get (2). In the second part of this paper we prove Theorem 1.1 by finding a functional equation satisfied by both sides of another equation equivalent to (6), see Theorem 4.1. Finding a combinatorial proof of Theorem 1.1 is still open. Our combinatorial model for (2) is naturally extended to a model for (6), and letting $k \rightarrow \infty$ as in the proof of (3), we obtain JTP. For this reason, Theorem 1.1 is a "finite version" of JTP.

\subsection{T-fractions}

Notice that we have the factor $\left(\left(\begin{array}{c}2 n \\ n-k\end{array}\right)-\left(\begin{array}{c}2 n \\ n-k-1\end{array}\right)\right)$ in the sums of (1), (2) and (6). This will be explained by the link between $S$-fractions and $T$-fractions (see Lemma 2.5). The family of $T$-fractions is the natural form of two-points Padé approximants $\left[\mathrm{CPV}^{+}\right.$08], they also appear occasionally in combinatorics [RV96] but much less than $S$-fractions or $J$-fractions. We use here a particular kind of $T$-fractions as in Definition 2 below. They are related with weighted Schröder paths, so that our main result can be stated as an exact formula for a certain weight sum of Schröder paths. The main property of $T$-fractions we use to prove the result is the fact that they satisfy certain functional equations.

\section{Preliminaries}

\subsection{Penaud's decomposition}

Definition 1. A Schröder path of length $2 n$ is a path from $(0,0)$ to $(2 n, 0)$ in $\mathbb{N}^{2}$ with three kinds of steps: an up step $(1,1)$, a down step $(1,-1)$, and a horizontal step $(2,0)$. A Dyck path is a Schröder path without any horizontal step. A marked Schröder path is a Schröder path in which each up step and down step may be marked. Let $\mathcal{S}_{n}$ (resp. $\overline{\mathcal{S}}_{n}, \mathcal{D}_{n}, \overline{\mathcal{D}}_{n}$ ) denote the set of Schröder paths (resp. marked Schröder paths, Dyck paths, marked Dyck paths) of length $2 n$. Let $\overline{\mathcal{D}}_{n}^{*}$ denote the subset of $\overline{\mathcal{D}}_{n}$ consisting of the marked Dyck paths without any peak, i.e. an up step immediately followed by a down step, consisting two marked steps.

Note that $\mathcal{D}_{n} \subset \mathcal{S}_{n}$ and $\mathcal{D}_{n}^{*} \subset \mathcal{S}_{n}^{*}$. We will also consider that $\mathcal{D}_{n} \subset \mathcal{D}_{n}^{*}$ and $\mathcal{S}_{n} \subset \mathcal{S}_{n}^{*}$ by identifying a Schröder path with a marked Schröder path without any marked step.

Given sequences $\mathcal{A}=\left(a_{1}, a_{2}, \ldots\right), \mathcal{B}=\left(b_{1}, b_{2}, \ldots\right)$ and a marked Schröder path $p$, we define the weight $\mathrm{wt}(p ; A, B)$ to be the product of $a_{h}$ (resp. $b_{h}$ ) for each unmarked up step (resp. unmarked down step) between height $h$ and $h-1$, and -1 for each horizontal step (hence each marked step has weight 1). Since $\mathcal{S}_{n} \subset \mathcal{S}_{n}^{*}$, the weight is also defined for a Schröder path.

By definition, it is easy to see that

$$
\sum_{p \in \mathcal{D}_{n}} \operatorname{wt}(p ; \mathcal{A}, \mathcal{B})=\sum_{p \in \overline{\mathcal{D}}_{n}} \operatorname{wt}(p ; \mathcal{A}-1, \mathcal{B}-1), \quad \sum_{p \in \mathcal{S}_{k}} \operatorname{wt}(p ; \mathcal{A}, \mathcal{B})=\sum_{p \in \overline{\mathcal{S}}_{k}} \operatorname{wt}(p ; \mathcal{A}-1, \mathcal{B}-1),
$$

where $\mathcal{A}-1$ means the sequence $\left(a_{1}-1, a_{2}-1, \ldots\right)$.

Now a generalized version of Penaud's decomposition can be describes as follows. Here, a Dyck prefix is a path in $\mathbb{N}^{2}$ from the origin to any point consisting of up steps and down steps.

Proposition 2.1. Each $p \in \overline{\mathcal{D}}_{n}$ can be uniquely decomposed into $\left(L, p^{\prime}\right)$ where $L$ is a Dyck prefix of length $2 n$ ending at height $2 k$ and $p^{\prime} \in \overline{\mathcal{D}}_{k}^{*}$ for some $k$. Moreover, for any sequences $\mathcal{A}$ and $\mathcal{B}$, we have $\operatorname{wt}(p ; \mathcal{A}, \mathcal{B})=\operatorname{wt}\left(p^{\prime} ; \mathcal{A}, \mathcal{B}\right)$. 
It is well known that the number of Dyck prefixes of length $2 n$ ending at height $2 k$ is equal to $\left(\begin{array}{c}2 n \\ n-k\end{array}\right)-$ $\left(\begin{array}{c}2 n \\ n-k-1\end{array}\right)$. Thus, from Proposition 2.1, we obtain

$$
\sum_{p \in \overline{\mathcal{D}}_{n}} \operatorname{wt}(p ; \mathcal{A}, \mathcal{B})=\sum_{k=0}^{n}\left(\left(\begin{array}{c}
2 n \\
n-k
\end{array}\right)-\left(\begin{array}{c}
2 n \\
n-k-1
\end{array}\right)\right) \sum_{p \in \overline{\mathcal{D}}_{k}^{*}} \mathrm{wt}(p ; \mathcal{A}, \mathcal{B})
$$

By (7) and (8), we get the following proposition.

Proposition 2.2. For any sequences $\mathcal{A}$ and $\mathcal{B}$, we have

$$
\sum_{p \in \mathcal{D}_{n}} \operatorname{wt}(p ; \mathcal{A}, \mathcal{B})=\sum_{k=0}^{n}\left(\left(\begin{array}{c}
2 n \\
n-k
\end{array}\right)-\left(\begin{array}{c}
2 n \\
n-k-1
\end{array}\right)\right) \sum_{p \in \overline{\mathcal{D}}_{k}^{*}} \mathrm{wt}(p ; \mathcal{A}-1, \mathcal{B}-1)
$$

On the other hand, by canceling a horizontal step and a peak with two marked steps, we obtain

$$
\sum_{p \in \overline{\mathcal{S}}_{k}} \mathrm{wt}(p ; \mathcal{A}, \mathcal{B})=\sum_{p \in \overline{\mathcal{D}}_{k}^{*}} \mathrm{wt}(p ; \mathcal{A}, \mathcal{B}) .
$$

By Proposition 2.2, (9) and (7), we get the following proposition.

Proposition 2.3. For any sequences $A$ and $B$, we have

$$
\sum_{p \in \mathcal{D}_{n}} \mathrm{wt}(p ; \mathcal{A}, \mathcal{B})=\sum_{k=0}^{n}\left(\left(\begin{array}{c}
2 n \\
n-k
\end{array}\right)-\left(\begin{array}{c}
2 n \\
n-k-1
\end{array}\right)\right) \sum_{p \in \mathcal{S}_{k}} \mathrm{wt}(p ; \mathcal{A}, \mathcal{B})
$$

\section{$2.2 T$-fractions and $S$-fractions}

We will use the space-saving notation for continued fractions:

$$
\frac{a_{0}}{b_{0}}-\frac{a_{1}}{b_{1}}-\frac{a_{2}}{b_{2}}-\cdots=\frac{a_{0}}{b_{0}-\frac{a_{1}}{b_{1}-\frac{a_{2}}{b_{2}-} \cdot}}
$$

Definition 2. To any sequence $\lambda=\left\{\lambda_{n}\right\}_{n \geq 1}$, we associate the $S$-fraction $S_{\lambda}(z)$ and the $T$-fraction $T_{\lambda}(z)$ :

$$
S_{\lambda}(z)=\frac{1}{1}-\frac{\lambda_{1} z}{1}-\frac{\lambda_{2} z}{1}-\frac{\lambda_{3} z}{1}-\ldots, \quad T_{\lambda}(z)=\frac{1}{1+z}-\frac{\lambda_{1} z}{1+z}-\frac{\lambda_{2} z}{1+z}-\frac{\lambda_{3} z}{1+z}-\ldots
$$

The combinatorial interpretation of $S$-fractions in terms of weighted Dyck paths is widely known, but the analogous result for $T$-fractions is not as common.

Lemma 2.4. Let $\mathcal{A}=\left(a_{1}, a_{2}, \ldots\right), \mathcal{B}=\left(b_{1}, b_{2}, \ldots\right)$ be two sequences and $\lambda_{h}=a_{h} b_{h}$. Then

$$
S_{\lambda}(z)=\sum_{n=0}^{\infty} z^{n} \sum_{p \in \mathcal{D}_{n}} \mathrm{wt}(p ; \mathcal{A}, \mathcal{B}), \quad T_{\lambda}(z)=\sum_{n=0}^{\infty} z^{n} \sum_{p \in \mathcal{S}_{n}} \operatorname{wt}(p ; \mathcal{A}, \mathcal{B}) .
$$


Proof. This can be proved by a classical method, see for example [GJ04].

By Lemma 2.4 and Proposition 2.3, we obtain the following lemma which gives a relation between the coefficients of an $S$-fraction and a $T$-fraction. This is a key step in our proofs of the Touchard-Riordan-like formulas.

Lemma 2.5. Given a sequence $\lambda=\left\{\lambda_{n}\right\}_{n \geq 1}$, we define $\mu=\left\{\mu_{n}\right\}_{n \geq 0}$ and $\nu=\left\{\nu_{n}\right\}_{n \geq 0}$ such that:

$$
\sum_{n=0}^{\infty} \mu_{n} z^{n}=S_{\lambda}(z), \quad \sum_{n=0}^{\infty} \nu_{n} z^{n}=T_{\lambda}(z)
$$

Then for any $n \geq 0$ we have the relation $\mu_{n}=\sum_{k=0}^{n}\left(\left(\begin{array}{c}2 n \\ n-k\end{array}\right)-\left(\begin{array}{c}2 n \\ n-k-1\end{array}\right)\right) \nu_{k}$.

\section{Combinatorics on weighted Dyck paths}

\subsection{A combinatorial proof of (2)}

From now on, we will fix two sequences

$$
\mathcal{U}=\left([1]_{q},[2]_{q}, \ldots\right), \quad \mathcal{V}=\left(1-q, 1-q^{2}, \ldots\right)
$$

By Lemma 2.4, Proposition 2.2, and the fact that $\operatorname{wt}(p ; \mathcal{U}, \mathcal{U})=\operatorname{wt}(p ; \mathcal{V}, \mathcal{V}) /(1-q)^{2 n}$ for all $p \in \mathcal{D}_{n}$, the left hand side of (2) is equal to

$$
\sum_{p \in \mathcal{D}_{n}} \operatorname{wt}(p ; \mathcal{U}, \mathcal{U})=\frac{1}{(1-q)^{2 n}} \sum_{k=0}^{n}\left(\left(\begin{array}{c}
2 n \\
n-k
\end{array}\right)-\left(\begin{array}{c}
2 n \\
n-k-1
\end{array}\right)\right) \sum_{p \in \overline{\mathcal{D}}_{k}^{*}} \mathrm{wt}(p ; \mathcal{V}-1, \mathcal{V}-1)
$$

Thus in order to get (2) it is sufficient to show the following identity:

$$
\sum_{p \in \overline{\mathcal{D}}_{k}^{*}} \operatorname{wt}(p ; \mathcal{V}-1, \mathcal{V}-1)=q^{k(k+1)} \sum_{i=-k}^{k}(-q)^{-i^{2}}
$$

We introduce some terminologies. We denote by $\delta_{k}$ the staircase partition $(k, k-1, \ldots, 1)$.

Definition 3. A $\delta_{k}$-configuration is a pair $(\lambda, A)$ of a partition $\lambda \subset \delta_{k-1}$ and a set $A$ of arrows occupying a whole row or a whole column of $\delta_{k} / \lambda$ such that no inner corner of $\delta_{k} / \lambda$ is occupied by two arrows. Here, by an inner corner we mean a cell $c \in \delta_{k} / \lambda$ such that $\lambda \cup c$ is a partition. The length of an arrow is the number of cells occupied by the arrow. Let $\mathfrak{G}_{k}$ denote the set of $\delta_{k}$-configurations. We define the $q$-weight of a $\delta_{k}$-configuration $\Delta=(\lambda, A)$ to be

$$
\mathrm{wt}_{q}(\Delta)=(-1)^{|A|} q^{2|\lambda|+\|A\|},
$$

where $\|A\|$ is the sum of arrow lengths. 

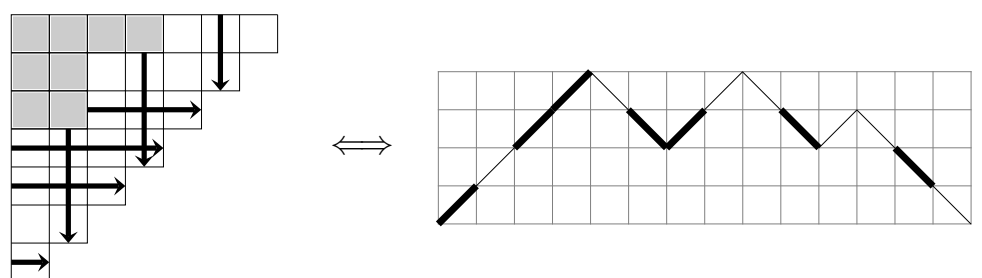

Figure 1: A $\delta_{k}$-configuration and the corresponding marked Dyck path, where marked steps are the thicker steps.
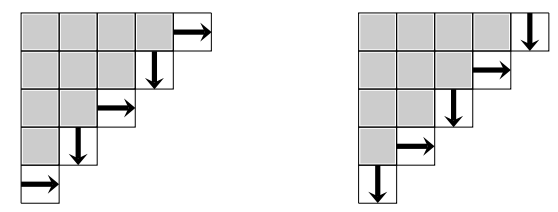

Figure 2: The two fixed points in $\mathfrak{G}_{5}$ which will not be sent to $\mathfrak{G}_{4}$.

For example, the $q$-weight of the $\delta_{k}$-configuration in Figure 1 is $(-1)^{7} q^{2 \cdot 8+1+3+4+3+3+3+2}$.

There is a simple bijection between $\overline{\mathcal{D}}_{k}^{*}$ and $\mathfrak{G}_{k}$ as follows. For $\Delta=(\lambda, A) \in \mathfrak{G}_{k}$, the north-west border of $\delta_{k} / \lambda$ defines a marked Dyck path of length $2 k$ where the marked steps correspond to the segments on the border with an arrow, see Figure 1. Moreover, if $p \in \overline{\mathcal{D}}_{k}^{*}$ corresponds to $\Delta \in \mathfrak{G}_{k}$, one can show that $\mathrm{wt}(p ; \mathcal{V}-1, \mathcal{V}-1)=q^{k(k+1)} \mathrm{wt}_{q^{-1}}(\Delta)$. Thus we obtain the following lemma.

Lemma 3.1. For any nonnegative integer $k$, we have

$$
\sum_{p \in \overline{\mathcal{D}}_{k}^{*}} \operatorname{wt}(p ; \mathcal{V}-1, \mathcal{V}-1)=q^{k(k+1)} \sum_{\Delta \in \mathfrak{G}_{k}} \mathrm{wt}_{q^{-1}}(\Delta)
$$

We prove the proposition below by constructing a weight-preserving-sign-reversing involution on $\mathfrak{G}_{k}$. More precisely, we construct such an involution on $\mathfrak{G}_{k}$ such that the fixed point set is in weight-preserving bijection with $\mathfrak{G}_{k-1}$ except the two fixed points $\Delta=(\lambda, A)$ with $\lambda=\delta_{k-1}$ and $A$ consisting of $n$ arrows alternating as shown in Figure 2. Thus our involution implies

$$
\sum_{\Delta \in \mathfrak{G}_{k}} \mathrm{wt}_{q}(\Delta)=\sum_{\Delta \in \mathfrak{G}_{k-1}} \mathrm{wt}_{q}(\Delta)+2(-q)^{k^{2}} .
$$

The key idea of the involution is "moving" the arrows upwards or to the left to decrease the arrow length by 1 . We omit the details.

Proposition 3.2. For a nonnegative integer $k$, we have

$$
\sum_{\Delta \in \mathfrak{G}_{k}} \mathrm{wt}_{q}(\Delta)=\sum_{i=-k}^{k}(-q)^{i^{2}}
$$

By Lemma 3.1 and Proposition 3.2 we obtain (15), thus completing the combinatorial proof of (2). 


\subsection{Limiting case $k \rightarrow \infty$ and a connection with JTP}

For $\Delta=(\lambda, A) \in \mathfrak{G}_{k}$, if there is an arrow coming from a row or a column of $\lambda$, one can easily see that $q^{k}$ divides $\mathrm{wt}_{q}(\Delta)$. In other words, if $\mathrm{wt}_{q}(\Delta)$ is not divisible by $q^{k}$, then the partition, the horizontal arrows and the vertical arrows are completely separated. Thus we can freely choose a partition, vertical arrows of distinct length and horizontal arrows of distinct length. This argument gives us the following.

Proposition 3.3. For any nonnegative integer $k$, we have

$$
\sum_{\Delta \in \mathfrak{G}_{k}} \mathrm{wt}_{q}(\Delta) \equiv \prod_{i \geq 1} \frac{1}{1-q^{2 i}} \prod_{i \geq 1}\left(1-q^{i}\right) \prod_{i \geq 1}\left(1-q^{i}\right)=\prod_{i \geq 1} \frac{1-q^{i}}{1+q^{i}} \bmod q^{k}
$$

Letting $k \rightarrow \infty$ in Propositions 3.2 and 3.3, we get (3).

Now we define the $(y, q)$-weight of a $\delta_{k}$-configuration $\Delta=(\lambda, A)$ to be

$$
\mathrm{wt}_{y, q}(\Delta)=(-1)^{|A|} q^{2|\lambda|+\|A\|}(-y)^{o h(A)-o v(A)},
$$

where $o h(A)$ (resp. $o v(A)$ ) is the number of odd-length horizontal (resp. vertical) arrows in $A$. For example, the $(y, q)$-weight of the $\delta_{k}$-configuration in Figure 1 is $(-1)^{7} q^{2 \cdot 8+1+3+4+3+3+3+2}(-y)^{3-2}$.

The proof of the lemma below is similar to that of Lemma 3.1. Here we define

$$
\mathcal{J}=\left(1+y q, 1-q^{2}, 1+y q^{3}, 1-q^{4}, \ldots\right), \quad \mathcal{J}^{\prime}=\left(1+y^{-1} q, 1-q^{2}, 1+y^{-1} q^{3}, 1-q^{4}, \ldots\right) .
$$

Lemma 3.4. For any nonnegative integer $k$, we have

$$
\sum_{p \in \overline{\mathcal{D}}_{k}^{*}} \mathrm{wt}\left(p ; \mathcal{J}-1, \mathcal{J}^{\prime}-1\right)=q^{k(k+1)} \sum_{\Delta \in \mathfrak{G}_{k}} \mathrm{wt}_{y, q^{-1}}(\Delta) .
$$

By the same argument as in the proof of Proposition 3.3 together with

$$
\begin{array}{r}
\prod_{i \geq 1} \frac{1}{1-q^{2 i}} \prod_{i \geq 1}\left(1-q^{2 i}\right)\left(1+y q^{2 i-1}\right) \prod_{i \geq 1}\left(1-q^{2 i}\right)\left(1+y^{-1} q^{2 i-1}\right) \\
=\prod_{i \geq 1}\left(1-q^{2 i}\right)\left(1+y q^{2 i-1}\right)\left(1+y^{-1} q^{2 i-1}\right)
\end{array}
$$

we obtain the following.

Proposition 3.5. For any nonnegative integer $k$, we have

$$
\sum_{\Delta \in \mathfrak{G}_{k}} \mathrm{wt}_{y, q}(\Delta) \equiv \prod_{i \geq 1}\left(1-q^{2 i}\right)\left(1+y q^{2 i-1}\right)\left(1+y^{-1} q^{2 i-1}\right) \quad \bmod q^{k} .
$$

Since the right hand side of (16) is one side of JTP, it is natural to guess

$$
\sum_{\Delta \in \mathfrak{G}_{k}} \mathrm{wt}_{y, q}(\Delta)=\sum_{i=-k}^{k} y^{i} q^{i^{2}}
$$


Using Lemma 3.4 and the same argument as in (14), one can see that (17) is equivalent to Theorem 1.1. This is in fact the way the authors first discovered Theorem 1.1. Notice also that by Lemma 3.4, (9) and (7) in this order, we have

$q^{k(k+1)} \sum_{\Delta \in \mathfrak{G}_{k}} \mathrm{wt}_{y, q^{-1}}(\Delta)=\sum_{p \in \overline{\mathcal{D}}_{k}^{*}} \mathrm{wt}\left(p ; \mathcal{J}-1, \mathcal{J}^{\prime}-1\right)=\sum_{p \in \overline{\mathcal{S}}_{k}} \mathrm{wt}\left(p ; \mathcal{J}-1, \mathcal{J}^{\prime}-1\right)=\sum_{p \in \mathcal{S}_{k}} \operatorname{wt}\left(p ; \mathcal{J}, \mathcal{J}^{\prime}\right)$.

Thus (17) is also equivalent to

$$
\sum_{p \in \mathcal{S}_{k}} \operatorname{wt}\left(p ; \mathcal{J}, \mathcal{J}^{\prime}\right)=\sum_{j=-k}^{k} y^{j} q^{k(k+1)-j^{2}}
$$

We will prove this in the next section.

\subsection{Generalized q-secant numbers}

For two nonnegative integers $a$ and $b$, we define

$$
E_{n}^{a, b}(q)=\left[z^{n}\right]\left(\frac{1}{1}-\frac{[a+1]_{q}[b+1]_{q} z}{1}-\frac{[a+2]_{q}[b+2]_{q} z}{1}-\cdots\right) .
$$

Then $E_{n}^{0,0}(q)$ is a $q$-secant number and $E_{n}^{0,1}(q)$ is a $q$-tangent number, see [JV10].

By (2), we have

$$
E_{n}^{0,0}(q)=\frac{1}{(1-q)^{2 n}} \sum_{k=0}^{n}\left(\left(\begin{array}{c}
2 n \\
n-k
\end{array}\right)-\left(\begin{array}{c}
2 n \\
n-k-1
\end{array}\right)\right) q^{k(k+1)} T_{k}\left(q^{-1}\right)
$$

where $T_{k}(q)=\sum_{i=-k}^{k}(-q)^{i^{2}}$. Note that by (3) we have

$$
\lim _{k \rightarrow \infty} T_{k}(q)=\frac{(q ; q)_{\infty}}{(-q ; q)_{\infty}}
$$

where we use the usual notation $(a ; q)_{n}=(1-a)(1-a q) \cdots\left(1-a q^{n-1}\right)$ and $(a ; q)_{\infty}=(1-a)(1-$ $a q) \cdots$. We generalize this as follows.

Theorem 3.6. For nonnegative integers a and $b$, there is a family $\left\{T_{k}^{a, b}(q)\right\}_{k \geq 0}$ of polynomials in $q$ such that

$$
E_{n}^{a, b}(q)=\frac{1}{(1-q)^{2 n}} \sum_{k=0}^{n}\left(\left(\begin{array}{c}
2 n \\
n-k
\end{array}\right)-\left(\begin{array}{c}
2 n \\
n-k-1
\end{array}\right)\right) q^{k(k+1+a+b)} T_{k}^{a, b}\left(q^{-1}\right)
$$

Moreover, for all $k \geq 0$, we have

$$
T_{k}^{a, b}(q) \equiv \frac{1}{(q ; q)_{a}(q ; q)_{b}} \cdot \frac{(q ; q)_{\infty}}{(-q ; q)_{\infty}} \bmod q^{k}
$$

which implies

$$
\lim _{k \rightarrow \infty} T_{k}^{a, b}(q)=\frac{1}{(q ; q)_{a}(q ; q)_{b}} \cdot \frac{(q ; q)_{\infty}}{(-q ; q)_{\infty}}
$$




\section{The finite version of Jacobi's triple product and consequences}

\subsection{Main result}

By Lemma 2.5, Theorem 1.1 is equivalent to the following theorem. This is also equivalent to (17) and (18), and as we have seen, these identities give JTP when $k$ tends to infinity.

Theorem 4.1. There holds

$$
\sum_{k=0}^{\infty} z^{k} \sum_{j=-k}^{k} y^{j} q^{k(k+1)-j^{2}}=\frac{1}{1+z}-\frac{(1+q y)\left(1+q y^{-1}\right) z}{1+z}-\frac{\left(1-q^{2}\right)^{2} z}{1+z}-\ldots
$$

where the continued fraction being $T_{\lambda}(z)$ with $\lambda_{n}=\left(1+q^{n} y\right)\left(1+q^{n} y^{-1}\right)$ for odd $n$ and $\lambda_{n}=\left(1-q^{n}\right)^{2}$ for even $n$.

Proof. We show that both sides satisfy a common functional equation. Indeed, if $H(z)$ is the left-hand side of (19) and $c_{j, k}(z)=z^{k} y^{j} q^{k(k+1)-j^{2}}$, we have

$$
H(z)=\sum_{j, k \in \mathbb{Z}, k \geq|j|} c_{j, k}(z), \quad c_{j, k+1}(z)=z q^{2} c_{j, k}\left(z q^{2}\right),
$$

and it follows that

$$
H(z)-z q^{2} H\left(z q^{2}\right)=\sum_{j \in \mathbb{Z}} c_{j,|j|}(z)=\frac{1}{1-y q z}+\frac{1}{1-y^{-1} q z}-1 .
$$

To show the last equality, note that when $k=|j|$ the term $k^{2}$ cancels with $-j^{2}$ in $c_{j, k}(z)$ and splitting the $j$-sum according to the sign of $j$ gives two geometric series. So $H(z)$ is the unique formal power series satisfying the functional equation:

$$
H(z)=\frac{1}{1-y q z}+\frac{1}{1-y^{-1} q z}-1+z q^{2} H\left(z q^{2}\right) .
$$

Uniqueness comes from the fact that by iterating (22), we can get more and more terms in the expansion of $H(z)$. It remains only to show that the continued fraction in the right-hand side of (19) satisfies the same functional equation, which is done in a separate lemma below.

Lemma 4.2. Let $\lambda$ as in Theorem 4.1, then we have

$$
T_{\lambda}(z)=\frac{1}{1-y q z}+\frac{1}{1-y^{-1} q z}-1+z q^{2} T_{\lambda}\left(z q^{2}\right) .
$$

Proof. We will identify $2 \times 2$-matrices and Möbius transformation in the usual way:

$$
\left(\begin{array}{ll}
a & b \\
c & d
\end{array}\right)[X]=\frac{a X+b}{c X+d}
$$


i.e. we use a bracket notation for the evaluation of a Möbius transformation. A continued fraction can be obtained by iterating such transformations. In the present case, we have

$$
\frac{1}{1+z-\frac{(1+w q y)\left(1+w q y^{-1}\right) z}{1+z-\left(1-w q^{2}\right)^{2} z X}}=\frac{z\left(1-w q^{2}\right)^{2} X-(1+z)}{z(1+z)\left(1-w q^{2}\right)^{2} X+(1+w q y)\left(1+w q y^{-1}\right) z-(1+z)^{2}},
$$

so we can introduce the matrix

$$
M(w, z)=\left(\begin{array}{cc}
z\left(1-w q^{2}\right)^{2} & -1-z \\
z(1+z)\left(1-w q^{2}\right)^{2} & (1+w q y)\left(1+w q y^{-1}\right) z-(1+z)^{2}
\end{array}\right)
$$

and we have

$$
T_{\lambda}(z)=\prod_{n=0}^{\infty} M\left(q^{2 n}, z\right) .
$$

The partial products are just the convergents of the continued fraction. More precisely, the infinite product is convergent in the following sense: the partial products are Möbius transformations, and these converge pointwise to the formal power series in the left-hand side of (27).

Let $S$ be the matrix

$$
S=\left(\begin{array}{cc}
z q^{2} & \frac{1}{1-z q y}+\frac{1}{1-z q y^{-1}}-1 \\
0 & 1
\end{array}\right)
$$

The functional equation that we want to prove can be written:

$$
\prod_{n=0}^{\infty} M\left(q^{2 n}, z\right)=S \prod_{n=0}^{\infty} M\left(q^{2 n}, z q^{2}\right)
$$

By examining the previous equation, it is natural to introduce a matrix $\Omega_{n}$ by

$$
\Omega_{n}=M\left(q^{2 n}, z\right)^{-1} \cdots M(1, z)^{-1} S M\left(1, z q^{2}\right) \cdots M\left(q^{2 n}, z q^{2}\right)
$$

where we understand that only even powers of $q$ appear within the dots. It can be calculated explicitly, as given in Lemma 4.3 below, so that we obtain:

$$
\Omega_{n}[0]=\frac{1-z^{2} q^{2}}{z q^{2 n+3}\left(2 q z-y-y^{-1}\right)+1-z^{2} q^{2}} .
$$

The important point is that from this closed form, we can check that $\Omega_{n}[0]$ is well-defined at $z=0$ (i.e. it has no pole at $z=0$ ). Let $w_{n}=\Omega_{n}[0]$, by definition of $\Omega_{n}$ we have:

$$
M(1, z) \cdots M\left(q^{2 n}, z\right)\left[w_{n}\right]=S M\left(1, z q^{2}\right) \cdots M\left(q^{2 n}, z q^{2}\right)[0],
$$

and at this point it remains only to let $n$ tend to infinity in (32) to prove (29), which was a rewriting of (23). The only subtlety is in the left-hand side, where we need the fact that $w_{n}$ is indeed a formal power series in $z$ (as opposed to a formal Laurent series) to take the limit. More precisely, one can show by a straightforward calculation that the left-hand side in (32) does not depend on $w_{n}$ up to a $O\left(z^{n+1}\right)$, and this is why we can take the limit $n \rightarrow \infty$. 
Lemma 4.3. The matrix $\Omega_{n}$ defined in (30) has the explicit form:

$$
\Omega_{n}=\frac{\left(\begin{array}{cc}
q^{2} z\left(2 q^{2 n+2}-z q^{2 n+3}\left(y+y^{-1}\right)+z^{2} q^{2}-1\right) & \begin{array}{c}
1-z^{2} q^{2} \\
\left(1-q^{2 n+2}\right)^{2}\left(z^{2} q^{2}-1\right) z q^{2}
\end{array} \\
z q^{2 n+3}\left(2 q z-y-y^{-1}\right)+1-z^{2} q^{2}
\end{array}\right)}{(1-y z q)\left(1-y^{-1} z q\right)} .
$$

Proof. Although calculations are quite cumbersome, there is a straightforward recursive verification of the given expression, using the relation, for $n \geq 1$,

$$
\Omega_{n}=M\left(q^{2 n}, z\right)^{-1} \Omega_{n-1} M\left(q^{2 n}, z q^{2}\right),
$$

where we define $\Omega_{0}=S$. There are 4 coefficients in $\Omega_{n}$, each appears as a sum of 4 terms when we expand the previous equation, and each of this term is a product of 3 coefficients of the matrices in (26) and (33). So there is a small "explosion" of the size of computations to perform. However, this is a verification that can be done with no particular cleverness, since expanding everything in (34) will clearly makes possible a term-by-term identification of both sides. We omit details and invite the unconvinced reader to use some computer algebra system for checking that the lemma is true.

\subsection{New Touchard-Riordan-like formulas}

From Theorem 4.1, we can derive a whole family of $S$-fractions having associated Touchard-Riordan formulas, as given in the theorem below. A very interesting property of these is that there are exponential generating functions linked with trigonometric functions. The theorem below is also a wide generalization of the result in (2), which is related with a $q$-analog of secant numbers having exponential generating function $\sec (z)$. Note that in the definition of $[n]_{q}=\left(1-q^{n}\right) /(1-q), n$ can be any number, not necessarily an integer.

Theorem 4.4. For any numbers $a$ and $b$, we define $\mu_{n}(a, b, q)$ by

$$
\sum_{n=0}^{\infty} \mu_{n}(a, b, q) z^{n}=S_{\lambda}(z), \quad \text { where } \quad \lambda_{n}= \begin{cases}{[n b+a]_{q}[n b-a]_{q}} & \text { if } n \text { is odd }, \\ {[n b]_{q}^{2}} & \text { if } n \text { is even. }\end{cases}
$$

Then we have

$$
\mu_{n}(a, b, q)=\frac{1}{(1-q)^{2 n}} \sum_{k=0}^{n}\left(\left(\begin{array}{c}
2 n \\
n-k
\end{array}\right)-\left(\begin{array}{c}
2 n \\
n-k-1
\end{array}\right)\right) \sum_{j=-k}^{k}(-1)^{j} q^{a j+b\left(k(k+1)-j^{2}\right)},
$$

and

$$
\sum_{n=0}^{\infty} \mu_{n}(a, b, 1) \frac{z^{2 n}}{(2 n) !}=\frac{\cos (a z)}{\cos (b z)}
$$

Proof. Consider the identity obtained by substituting $(y, q)$ with $\left(-q^{a}, q^{b}\right)$ in (19), and apply Lemma 2.5 to this $T$-fraction. This gives the desired formula for $(1-q)^{2 n} \mu_{n}(a, b, q)$.

It remains only to obtain the exponential generating function of $\mu_{n}(a, b, 1)$ as a ratio of cosines. Actually, this was essentially known by Stieltjes [Sti90] via analytical methods. It is also possible to prove this going through an addition formula satisfied by $\cos (a z) / \cos (b z)$, and using a theorem of Stieltjes and Rogers, see for example in [GJ04, Chapter 5] (this method is generally well-suited for trigonometric functions). 
Note that $\mu(a, b, q)$ is a polynomial in $q$ with nonnegative coefficients in the following situation: $a$ and $b$ are integers such that $0 \leq a<b$, and also (this is less obvious): $a$ and $b$ are half-integers satisfying the same inequalities. This implies that a function such as $\cos (z / 2) / \cos (3 z / 2)$ is the exponential generating function for a sequence of nonnegative integers. Thus Theorem 4.4 opens some problems, for a better understanding of these quantities $\mu_{n}(a, b, q)$. We can ask if there is a combinatorial interpretation from which both the ordinary generating function and the exponential one (for $q=1$ ) can be obtained. It would be quite remarkable to thus obtain the continued fraction on one side and the trigonometric function on the other side.

\section{Acknowledgements}

This work started when both authors were in Paris in the group of Sylvie Corteel. We thank her for discussions and encouragements.

\section{References}

[AB04] K. Alladi and A. Berkovich. New polynomial analogues of Jacobi's triple product and Lebesgue's identities. Adv. in Appl. Math., 32(4):801-824, 2004.

[Ber06] B.C. Berndt. Number theory in the spirit of Ramanujan. AMS, 2006.

$\left[\mathrm{CPV}^{+} 08\right]$ A. Cuyt, V. Petersen, B. Verdonk, H. Waadeland, and W.B. Jones. Handbook of continued fractions for special functions. Springer, 2008.

[GJ04] I.P. Goulden and D.M. Jackson. Combinatorial enumeration. Dover, 2004.

[JV10] M. Josuat-Vergès. A q-enumeration of alternating permutations. European J. Combin., 31(7):1892-1906, 2010.

[Rea79] R.C. Read. The chord intersection problem. Ann. New York Acad. Sci., 319(1):444-454, 1979.

[Rio75] J. Riordan. The distribution of crossings of chords joining pairs of $2 n$ points on a circle. Math. Comp., 29(129):215-222, 1975.

[RV96] E. Roblet and X.G. Viennot. Théorie combinatoire des T-fractions et approximants de Padé en deux points. Discrete Math., 153(1-3):271-288, 1996.

[Sch05] M. Schlosser. Abel-rothe type generalizations of jacobi's triple product identity. In M.E.H. Ismail and E. Koelink, editors, Theory and Applications of Special Functions, pages 383-400. Springer, 2005.

[Sti90] T.J. Stieltjes. Sur quelques intégrales définies et leur développement en fractions continues. Q. J. Math., 24:370-382, 1890.

[Tou52] J. Touchard. Sur un problème de configurations et sur les fractions continues. Canad. J. Math., 4(2):25, 1952.

[War05] S.O. Warnaar. $q$-hypergeometric proofs of polynomial analogues of the triple product identity, Lebesgue's identity and Euler's pentagonal number theorem. Ramanujan J., 8(4):467-474, 2005 . 